\title{
Diversidad floristica presente en turberas de musgo Sphagnum en la cuenca del río Mayer, comuna de O’Higgins, región de Aysén, Chile
}

\author{
Floristic diversity present in Sphagnum peatland bogs \\ in the Mayer river basin, O'Higgins commune, Aysén region, Chile
}

Erwin Domínguez ${ }^{1}$, María Paz Martínez ${ }^{1}$, Dagoberto Villarroel ${ }^{1}$ \& José Manuel Henríquez ${ }^{1}$

\section{Resumen}

Las turberas de Sphagnum son humedales de alto valor ambiental y son frecuentes en la Patagonia. En particular en la región de Aysén, los estudios sobre su diversidad aún son insuficientes $y$ parciales. En este trabajo se evalúa la variabilidad de la composición y abundancia florística, presente en cuatro turberas de musgo Sphagnum continentales, sin intervención humana. Se establecieron 40 subparcelas de $1 \mathrm{~m}^{2}$ de manera aleatoria, a fin de determinar diferencias entre ellas; se estimó el índice de diversidad de ShannonWiener (H'), el índice de Simpson (D), el índice exponencial de entropía de Shannon (exp H') y el índice inverso de Gini-Simpson (1/1- $\lambda$ '). Con los datos obtenidos se realizó la prueba de KruskalWallis con la corrección de Bonferroni, para detectar diferencias entre las turberas, debido a que los datos no mostraron distribución normal (prueba de Shapiro-Wilk). Además, se aplicó un análisis exploratorio de componentes principales (PCA) con el fin de inferir las relaciones existentes entre las especies con algún gradiente ecológico. Un total de 33 taxones, 21 familias y 27 géneros fueron identificados. Las especies más dominantes fueron: Sphagnum magellanicum (Brid.) y Empetrum rubrum Vahl ex Willd. El indice de diversidad de Shannon-Wiener (H') varió entre 1,47 a 0,96, el índice de Simpson (D) entre 0,48 a 0,30, el índice exponencial de entropía de Shannon (exp H') entre 4,46 a 2,66 y el índice inverso de Gini-Simpson (1/1- $\lambda$ ') entre 0,52 a 0,69 . El PCA sugiere la existencia de un gradiente florístico relacionado a la hidrología.

\section{Palabras clave:}

biodiversidad, turbera, musgo Sphagnum, Patagonia.

\section{Abstract}

Sphagnum peatland are wetlands of high environmental value and are frequent in Patagonia. In particular in the Aysén region, studies on its biodiversity are still insufficient and partial. In this work, the variability of the composition and floristic abundance is evaluated, present in four continental Sphagnum peatland bogs, without human intervention. 40 subplots of $1 \mathrm{~m}^{2}$ were established randomly in order to determine differences between them, the Shannon-Wiener diversity index $\left(\mathrm{H}^{\prime}\right)$ was estimated, the Simpson index (D), the exponential of Shannon's entropy index (exp H') and the inverse Gini-Simpson index $\left(1 / 1-\lambda{ }^{\prime}\right)$. With the data obtained, the Kruskal-Wallis test was performed with the Bonferroni correction, to detect differences between the peatland bogs, because the data did not show normal distribution (Shapiro-Wilk test). In addition, an exploratory principal components analysis (PCA) was applied in order to infer the existing relationships between species with some ecological gradient. A total of 33 taxa, 21 families, and 27 genera were identified. The most dominant species were: Sphagnum magellanicum (Brid.) and Empetrum rubrum Vahl ex Willd. The Shannon-Wiener diversity index

\footnotetext{
Instituto de Investigaciones Agropecuarias INIA §edominguez@inia.cl
} 
( $\left.H^{\prime}\right)$ varied between 1.47 to 0.96 , the Simpson index (D) between 0.48 to 0.30 , the exponential of Shannon's entropy index (exp H') between 4.46 to 2.66 and the inverse Gini-Simpson index (1/1$\lambda$ ') between 0.52 to 0.69 . The PCA suggests the existence of a floristic gradient related to hydrology.

\section{Key words:}

Biodiversity, Peat bog, Sphagnum moss, Patagonia.

\section{INTRODUCCIÓN}

Las turberas son hábitats de alto valor ambiental, ellas se encargan de la regulación, almacenamiento y purificación del agua, sustentan una biodiversidad única de plantas y animales. Además, juegan un papel esencial en el ciclo global del carbono en el contexto del cambio climático, al funcionar como sumideros de aquel elemento, ya que estos ambientes han almacenado entre 500 y 700 giga-toneladas de carbono atmosférico, equivalente al $30 \%$ del total del C orgánico global del suelo (Yu et al. 2011; Armstrong et al. 2015). Por otra parte, el Ministerio de Agricultura de Chile ha reconocido al musgo Sphagnum magellanicum (Brid.) como un recurso silvoagropecuario, creando el primer instrumento de regulación que tiene por finalidad proteger este recurso y mitigar los impactos ambientales, sociales y económicos en las zonas donde se cosecha esta especie, a través del Decreto Supremo $N^{\circ} 25$. Esto último implica la necesidad de recopilar información sobre la diversidad, ecología, distribución geográfica y la superficie de las turberas en las distintas regiones del país en donde crece el musgo $S$. magellanicum.

La vegetación de las turberas de la Región de Aysén del General Carlos Ibáñez del Campo ha sido poco estudiada. Entre las investigaciones destaca la caracterización florística de las turberas de Tortel, específicamente en la cuenca de los ríos Vagabundo, Barrancoso y en el desagüe del lago Quetru (Ramírez et al. 2007). Otro estudio realizado el año 2008, hace un exhaustivo levantamiento de las turberas en las cuencas de los ríos Baker y Pascua, identificando 28 plantas vasculares (Rodríguez et al. 2008). El mismo año se realiza un estudio que describe la vegetación de dos turberas, localizadas en las inmediaciones de: 1) laguna Pedro Aguirre Cerda al norte de Mañihuales, y 2) lago Vargas al sur de Cochrane; mientras que en la primera se encontró sólo 18 plantas vasculares, en la segunda fueron identificadas 23, todas nativas. De acuerdo con Teneb y colaboradores (2008), las diferencias florísticas halladas entre ambas turberas podrían deberse a gradientes de precipitación y a variables físico-químicas dadas por el origen geológico y la antigüedad de los valles, así como el horizonte de acumulación de turba y el pH del sustrato. García y Luebert (2008) describen la flora y vegetación en el río Mosco, al oriente de Villa O־Higgins, incluyendo algunos pequeños ambientes de turberas, no especificados. Álvarez y colaboradores (2010) reportaron una riqueza florística de 31 especies para una turbera de Sphagnum en un estudio en el archipiélago de los Chonos. Otro estudio realiza una descripción de las turberas de Sphagnum, pulvinadas y graminosas en la comuna de Tortel, describiendo la presencia de turberas subarbustivas dominadas por el ciprés enano (Lepidothamnus fonkii), el que presenta gran afinidad florística y ecológica con las turberas esfagnosas y al parecer representaría un estadio terminal de las turberas de Sphagnum magellanicum (San Martín et al. 2014). Sandoval y colaboradores (2016) describen y comparan atributos de la biodiversidad vegetal en las cuencas de los ríos Baker y Pascua, reportando 66 especies para las turberas de Sphagnum y un índice de diversidad de Shannon -Wiener de $\left(\mathrm{H}^{\prime}=2,038\right)$.

A pesar de los diferentes esfuerzos por evaluar las turberas, sólo un estudio florístico realizó un análisis sobre la magnitud de la diversidad (Sandoval et al. 2016), entendiendo que los índices de biodiversidad son indicadores para analizar los atributos estructurales y de composición de las comunidades biológicas (Noss, 1990; Maclaurin \& Sterelny, 2008) y estos sirven para establecer la relación entre la riqueza y la abundancia, y su modificación como resultado de actividades humanas (Moreno et al. 2011; Jost \& GonzálezOreja, 2012).

El medir la magnitud de la diversidad se justifica para resumir una gran cantidad de información en un solo valor, permitiendo hacer comparaciones rápidas. Considerando que se sabe poco sobre la biodiversidad florística en las turberas de Aysén, y que las últimas turberas de Sphagnum continentales, sin intervención humana, se encuentran ubicadas en la comuna de O'Higgins, 


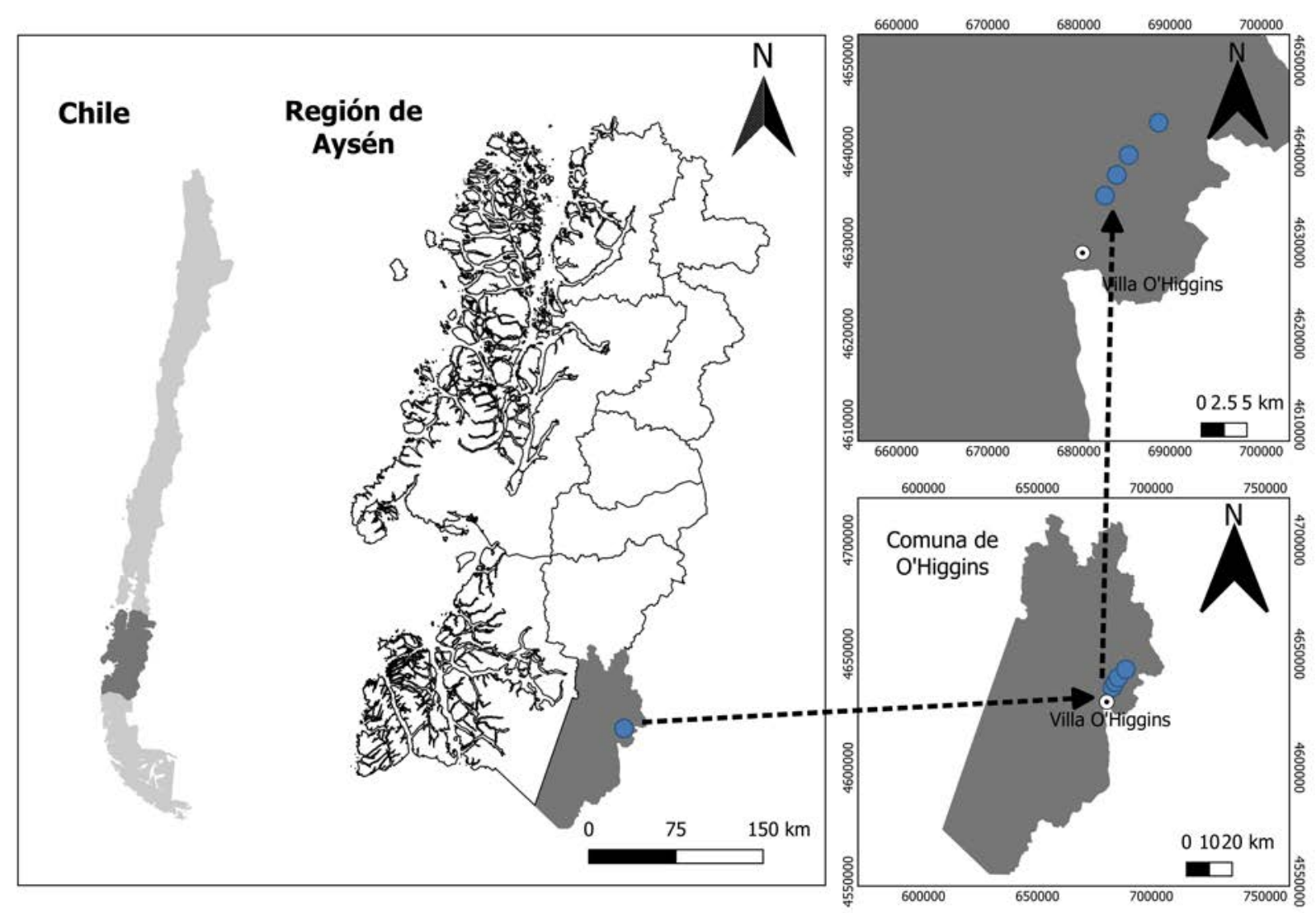

Fig. 1. Localización geográfica de las turberas estudiadas, en la comuna de O'Higgins.

se ha planteado la siguiente pregunta: ¿cuál es la diversidad presente dentro y entre las turberas de musgo Sphagnum en la comuna de O'Higgins? Para abordar esta interrogante se ha propuesto el siguiente objetivo: 1) evaluar la variabilidad de la composición y abundancia florística presente en turberas de musgo Sphagnum.

\section{MATERIAL Y MÉTODOS}

Área de estudio

Este estudio se realizó en la cuenca del río Mayer, en la comuna de O'Higgins, provincia Capitán Prat (región de Aysén) (Fig. 1). Esta comuna cuenta con una superficie de 4.338 ha de turberas de Sphagnum, las que representan un $28,5 \%$ del total regional. De las cuales 151 ha se encuentran en Áreas Silvestres Protegidasy corresponden al 3\% del territorio comunal (Villarroel et al. 2021). Posee un gradiente de elevación de 340 a 524 msnm El área ha sido clasificada dentro del bioclima templado hiperoceánico (Luebert \& Pliscoff, 2006). Según datos de la Dirección General de Aguas, la estación de Villa O'Higgins (48 $\left.28^{\prime} \mathrm{S}, \quad 72^{\circ} 33^{\prime} \mathrm{O}, 270 \mathrm{~m}\right)$ registra una precipitación media anual (1994-2008) de 758 $\mathrm{mm}$, mientras que la temperatura media anual para el mismo período es de $7,5^{\circ} \mathrm{C}$, no registrándose meses con temperaturas medias menores a $0^{\circ} \mathrm{C}$, excepto en años excepcionales (García \& Luebert, 2008). Las turberas se desarrollan sobre un tipo de suelo histosol. En cuanto a la geomorfología, la zona muestra claras evidencias de la acción de los glaciares en la modelación del paisaje.

\section{Diseño de muestreo}

Se georreferenciaron 4 turberas de la comuna de O'Higgins (región de Aysén) (Fig. 1), las cuales fueron muestreadas en los meses de primavera y verano entre 2018 y 2019. En cada sitio se 
Tabla 1. Ubicación de las turberas de Sphagnum no intervenidas en la comuna de O'Higgins, región de Aysén, Chile.

\begin{tabular}{|c|c|c|c|c|}
\hline \multirow[t]{2}{*}{ ID } & \multirow[t]{2}{*}{ Sitios } & \multicolumn{2}{|c|}{ Coordenadas } & \multirow{2}{*}{$\begin{array}{l}\text { Altura } \\
\text { msnm }\end{array}$} \\
\hline & & Latitud (S) & Longitud (O) & \\
\hline $\mathrm{T} 1$ & Sector Río Mayer & $48^{\circ} 24^{\prime} 37,3^{\prime \prime}$ & $72^{\circ} 31^{\prime} 41,7^{\prime \prime}$ & 340 \\
\hline $\mathrm{T} 2$ & Sector Río Mayer & $48^{\circ} 23^{\prime} 22,3^{\prime \prime}$ & $72^{\circ} 30^{\prime} 40,7^{\prime \prime}$ & 369 \\
\hline T3 & Sector Río Mayer & $48^{\circ} 22^{\prime} 12,3^{\prime \prime}$ & $72^{\circ} 29^{\prime} 43,7^{\prime \prime}$ & 469 \\
\hline $\mathrm{T} 4$ & Sector Río Mayer & $48^{\circ} 20^{\prime} 09,3^{\prime \prime}$ & $72^{\circ} 27^{\prime} 08,7^{\prime \prime}$ & 524 \\
\hline
\end{tabular}

estableció al azar 10 subparcelas de $1 \mathrm{~m}^{2}(0,5$ x 2 $\mathrm{m})$ en una parcela de una superficie de $1.000 \mathrm{~m}^{2}$ $(50$ x $20 \mathrm{~m})$ y se registró la presencia y cobertura de todas las especies vegetales, utilizando la escala de abundancia-cobertura de Braun-Blanquet (1979), modificada (van der Maarel, 2007). La posición geográfica y la altura de cada sitio se registraron usando un GPS Map 64x Garmin. Los trabajos de identificación de plantas se realizaron con claves de acuerdo a la Flora Patagónica de INTA (Correa, 1969, 1971, 1978, 1984, 1985, 1988, 1999). La nomenclatura de las especies y su hábito se basó en el Catálogo de Plantas Vasculares de Chile (Rodríguez \& Marticorena, 2019). El origen geográfico de las plantas vasculares se basa en la Flora Vascular del Cono Sur (http://www.darwin. edu.ar/) (Zuloaga et al. 2020).

\section{Análisis estadístico}

Con el fin de determinar los patrones emergentes de la diversidad florística entre las turberas de Sphagnum, se excluyó del análisis de los parámetros comunitarios y del cálculo de diversidad a la especie Sphagnum magellanicum. La complejidad de la comunidad vegetal presente en cada unidad muestral, se estimó a través del índice de Shannon-Wiener (H') (Shannon, 1948), Simpson (D) (Simpson, 1949), exponencial de entropía de Shannon (exp H') (Jost, 2006) y el inverso de GiniSimpson (1/1- $\lambda$ ') (Caso \& Gil, 1988; Jost, 2018), estos dos últimos modifican los clásicos índices de Shannon (Shannon, 1948) y Simpson (Simpson, 1949) respectivamente, transformándolos en números efectivos de especies, equivalente a una medida de verdadera diversidad (Jost, 2006, 2018). Con los datos obtenidos se realizó la prueba de Kruskal-Wallis con la corrección de Bonferroni, para detectar diferencias entre las turberas, debido a que los datos no mostraron distribución normal (prueba de Shapiro-Wilk). Con esta información se aplicó un análisis exploratorio de componentes principales (PCA) con el fin de inferir las relaciones existentes entre las especies con gradientes ecológicos. Todos los análisis, tanto univariados como multivariados, se realizaron en el programa PAST (Palaeontological Statistics) v4.0 (Hammer et al. 2001).

\section{RESULTADOS}

Se registró un total de 33 taxones nativos para las turberas de Sphagnum, pertenecientes a 21 familias y 27 géneros. Tres especímenes no pudieron ser identificados a nivel específico, sólo a nivel de género: dos briófitas (Polytrichadelphus sp. y Warnstorfia sp.), ambas de la clase Bryopsida, y un liquen (Cladonia sp.) de la clase Lecanoromycetes (Tabla 2). Las familias más diversas son: Cyperaceae (4 géneros/6 especies) y Ericaceae (2 géneros/3 especies). Los géneros que contienen dos especies son: Carex, Gaultheria, Nothofagus, Schoenus, Cladonia y Sphagnum y 21 géneros están representados por una especie. Las familias mejor representadas en relación con la frecuencia absoluta fueron: Sphagnaceae, Ericaceae, Cyperaceae, Cladoniaceae y Fagaceae (Fig. 2). Entre los hábitos de crecimiento de las plantas, destacan las Hierbas perennes con 15 especies, seguido por los Subarbustos con 6 especies, entre los cuales destaca Empetrum rubrum por su frecuencia y abundancia, y los Árboles están representados por 3 especies (Pilgerodendron uviferum, Nothofagus antarctica y Nothofagus betuloides (Tabla 2). Respecto al origen de las especies, 18 son nativas y 15 endémicas de acuerdo con la clasificación de la Flora Vascular del Cono Sur (Tabla 2). Las especies más importantes en términos de frecuencia son: 


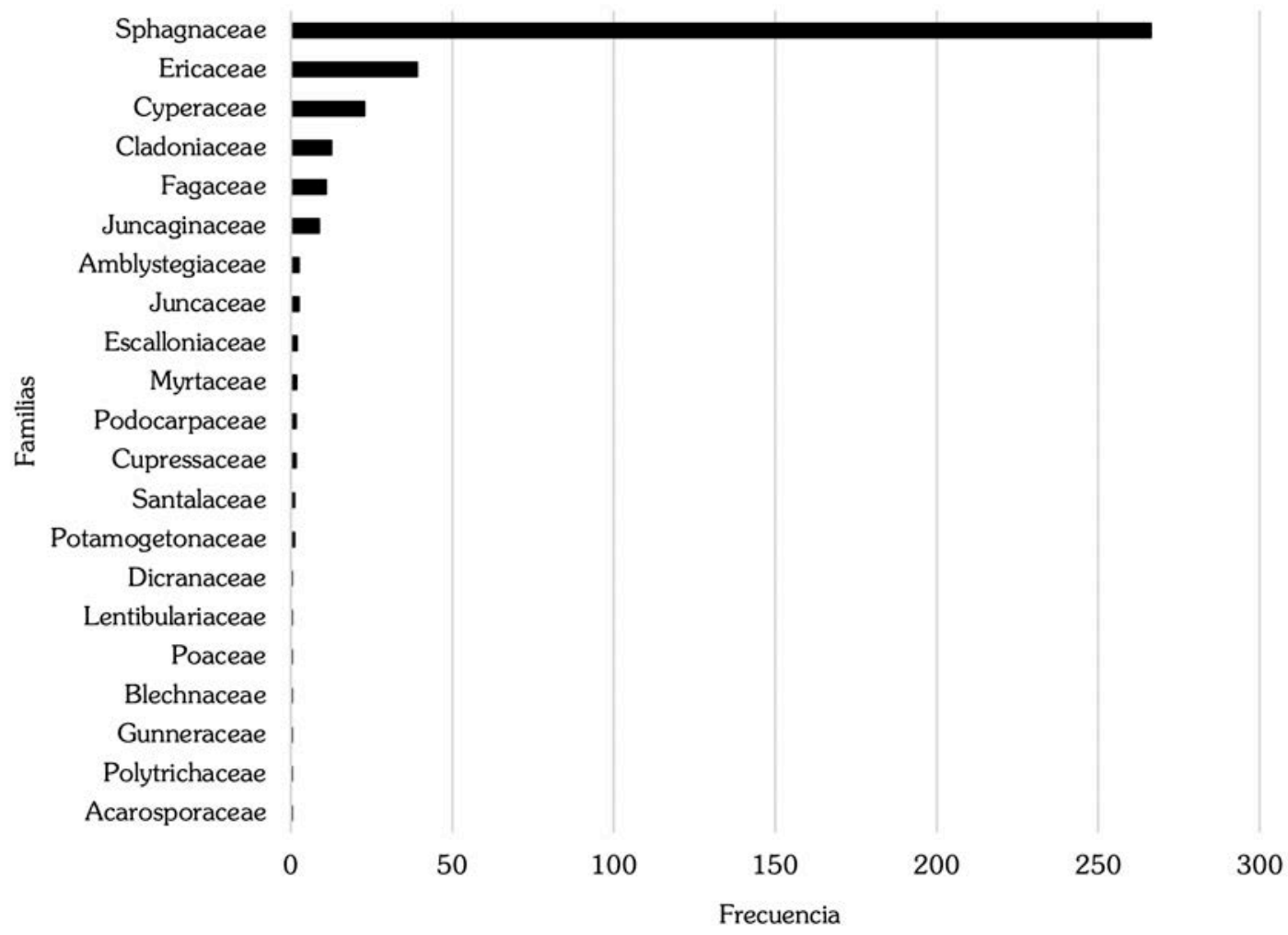

Fig. 2. Frecuencia absoluta de familias identificadas en las turberas de Sphagnum en la cuenca del río Mayer, comuna de O'Higgins.

Sphagnum magellanicum y Empetrum rubrum. Por otra parte, las especies raras en términos de cobertura y frecuencia, halladas en este estudio, son 21 (Fig. 2, Tabla 2).

El índice de Shannon-Wiener (H') reveló que las turberas T3 y T4 tienen una menor diversidad $(0,97$ y 0,96$)$ que T2 $(1,12)$, sin embargo, esta diferencia no es significativa entre ellas; mientras que T1 mostró una diversidad mayor $(1,47 ; \mathrm{p}<$ $0,05)$, pero un índice de Simpson (D) bajo $(0,31 ; p$ $<0,05)$, indicando que hay una especie dominante y una menor equitatividad (Tablas 2 y 3). Esto se refuerza con los índices exponencial de entropía de Shannon (exp H') e inverso de Gini-Simpson $\left(1 / 1-\lambda{ }^{\prime}\right)$ con los valores más altos para la turbera $\mathrm{T} 1$ respecto a las otras turberas (Tabla 3 ).

El PCA muestra la segregación de las especies en el plano formado por los dos componentes principales, que suman una varianza acumulada de $84 \%$. El componente 1 explica un $59 \%$ de variación y el componente 2 explica el $25 \%$ de variación. La mayoría de las especies se ubica en la intersección de los ejes, sin mayor diferenciación, esto estaría indicando que las turberas son homogéneas florísticamente entre sí. Sólo 6 especies se segregaron, tanto en el primero como en el segundo componente (Fig. 3). El componente 1 coincidiría con la profundidad de la napa freática según lo observado en terreno, siendo la especie indicadora más importante el musgo Sphagnum magellanicum, ubicado a la derecha del eje (X), especialmente asociado a las turberas T1 y T3. Por otra parte, la especie subarbustiva Empetrum rubrum se encuentra en sentido opuesto al gradiente hidrológico, es decir, en ambientes más secos. El componente 2 no está claro qué gradiente ambiental pueda estar representando, de acuerdo a su hábito de crecimiento Tetroncium magellanicum, una juncaginácea que se caracteriza por tener unas raíces profundas y crecer sobre sustratos con menor disponibilidad de oxígeno disuelto y una mayor conductividad eléctrica, en comparación con Carex magellanica, una ciperácea que se caracteriza por presentar un enraizamiento menos profundo, creciendo asociada al agua, en el borde de los cojines formados por el musgo Sphagnum. 
Tabla 2. Composición florística y cobertura promedio de las especies en las turberas de Sphagnum de la cuenca del río Mayer, en la comuna de O'Higgins. Hábito: $(A)=$ Árbol, (Ar) = Arbusto, (sAr) = Subarbusto,

$(\mathrm{Hp})=$ Hierba perenne, $(\mathrm{L})=$ Liquen, $(\mathrm{B})=$ Briófitaática. Origen: $(\mathrm{N})=$ Nativa,

$($ End) $=$ Endémica, T1, T2, T3 y T4 turberas y Cobertura promedio = Cob. $(\%)$.

\begin{tabular}{|c|c|c|c|c|c|c|c|c|}
\hline Clase & Familia & Especies & Hábito & Origen & $\begin{array}{l}\mathrm{T} 1 \\
\text { Cob. } \\
\text { (\%) }\end{array}$ & $\begin{array}{l}\text { T } 2 \\
\text { Cob. } \\
(\%)\end{array}$ & $\begin{array}{l}\text { T } 3 \\
\text { Cob. } \\
(\%)\end{array}$ & $\begin{array}{l}\text { T } 4 \\
\text { Cob. } \\
\text { (\%) }\end{array}$ \\
\hline Bryopsida & Sphagnaceae & $\begin{array}{l}\text { Sphagnum magellanicum } \\
\text { Brid. }\end{array}$ & B & $\mathrm{N}$ & 65,94 & 68,78 & 68,98 & 61 \\
\hline Magnoliopsida & Ericaceae & $\begin{array}{l}\text { Empetrum rubrum Vahl } \\
\text { ex Willd. }\end{array}$ & sAr & $\mathrm{N}$ & 4,55 & 9,4 & 7,4 & 13,1 \\
\hline Liliopsida & Cyperaceae & Carex magellanica Lam. & $\mathrm{Hp}$ & $\mathrm{N}$ & 2,98 & 1,2 & 9,1 & 2,35 \\
\hline Lecanoromycetes & Cladoniaceae & $\begin{array}{l}\text { Cladonia pycnoclada } \\
\text { (Pers.) Nyl. }\end{array}$ & $\mathrm{L}$ & $\mathrm{N}$ & 0,25 & 3,7 & 1,6 & 6,9 \\
\hline Magnoliopsida & Fagaceae & $\begin{array}{l}\text { Nothofagus antarctica } \\
\text { (G.Forst.) Oerst. }\end{array}$ & A & End & 3,63 & 2,8 & 3,2 & 0 \\
\hline Liliopsida & Juncaginaceae & $\begin{array}{l}\text { Tetroncium } \\
\text { magellanicum Willd. }\end{array}$ & $\mathrm{Hp}$ & End & 2,6 & 4,6 & 0,9 & 0,6 \\
\hline Magnoliopsida & Ericaceae & $\begin{array}{l}\text { Gaultheria antarctica } \\
\text { Hook.f. }\end{array}$ & sAr & End & 1,7 & 1,13 & 0,03 & 0,8 \\
\hline Bryopsida & Amblystegiaceae & Warnstorfia sp. & B & $\mathrm{N}$ & 0 & 0 & 2,5 & 0 \\
\hline Liliopsida & Cyperaceae & $\begin{array}{l}\text { Carex microglochin } \\
\text { Wahlenb }\end{array}$ & $\mathrm{Hp}$ & $\mathrm{N}$ & 1,36 & 0,2 & 0 & 0,9 \\
\hline Liliopsida & Cyperaceae & $\begin{array}{l}\text { Carpha schoenoides } \\
\text { Banks \& Sol. ex Hook. f. }\end{array}$ & $\mathrm{Hp}$ & End & 1,63 & 0,5 & 0 & 0 \\
\hline Liliopsida & Juncaceae & $\begin{array}{l}\text { Marsippospermum } \\
\text { grandiflorum (L.f.) Hook. } \\
\text { f. }\end{array}$ & $\mathrm{Hp}$ & $\mathrm{N}$ & 2 & 0 & 0 & 0 \\
\hline Magnoliopsida & Myrtaceae & $\begin{array}{l}\text { Myrteola nummularia } \\
\text { (Poiret) O.Berg }\end{array}$ & sAr & $\mathrm{N}$ & 1,45 & 0 & 0 & 0,2 \\
\hline Bryopsida & Sphagnaceae & $\begin{array}{l}\text { Sphagnum falcatulum } \\
\text { Besch. }\end{array}$ & B & $\mathrm{N}$ & 0 & 0 & 0 & 1,5 \\
\hline Pinopsida & Podocarpaceae & $\begin{array}{l}\text { Lepidothamnus fonkii } \\
\text { Phil. }\end{array}$ & sAr & End & 1,5 & 0 & 0 & 0 \\
\hline Pinopsida & Cupressaceae & $\begin{array}{l}\text { Pilgerodendron uviferum } \\
\text { (D.Don) Florin. }\end{array}$ & A & End & 1,5 & 0 & 0 & 0 \\
\hline Liliopsida & Cyperaceae & $\begin{array}{l}\text { Schoenoplectus } \\
\text { californicus (C.A.Mey.) } \\
\text { Soják }\end{array}$ & $\mathrm{Hp}$ & $\mathrm{N}$ & 1,5 & 0 & 0 & 0 \\
\hline Magnoliopsida & Escalloniaceae & Escallonia serrata $\mathrm{Sm}$. & $\mathrm{Ar}$ & End & 0 & 1,5 & 0 & 0 \\
\hline Magnoliopsida & Fagaceae & $\begin{array}{l}\text { Nothofagus betuloides } \\
\text { (Mirb.) Oerst. }\end{array}$ & A & End & 0 & 0,3 & 0 & 1 \\
\hline Magnoliopsida & Santalaceae & $\begin{array}{l}\text { Nanodea muscosa Banks } \\
\text { ex C.F.Gaertn. }\end{array}$ & $\mathrm{Hp}$ & End & 0,43 & 0,15 & 0,5 & 0,05 \\
\hline Magnoliopsida & Ericaceae & $\begin{array}{l}\text { Gaultheria mucronata } \\
\text { (L.f.) Hook. et Arn. }\end{array}$ & sAr & End & 0 & 0 & 1,03 & 0 \\
\hline Magnoliopsida & Potamogetonaceae & $\begin{array}{l}\text { Potamogeton linguatus } \\
\text { Hagstr. }\end{array}$ & $\mathrm{Hp}$ & End & 0 & 0 & 1,03 & 0 \\
\hline
\end{tabular}


Continuación Tabla 2

\begin{tabular}{|c|c|c|c|c|c|c|c|c|}
\hline Clase & Familia & Especies & Hábito & Origen & $\begin{array}{l}\text { T } 1 \\
\text { Cob. } \\
(\%)\end{array}$ & $\begin{array}{c}\text { T } 2 \\
\text { Cob. } \\
(\%)\end{array}$ & $\begin{array}{l}\text { T } 3 \\
\text { Cob. } \\
(\%)\end{array}$ & $\begin{array}{c}\text { T } 4 \\
\text { Cob. } \\
(\%)\end{array}$ \\
\hline Liliopsida & Cyperaceae & $\begin{array}{l}\text { Schoenus antarcticus } \\
\text { (Hook.f.) Dusén }\end{array}$ & $\mathrm{Hp}$ & End & 0,5 & 0,2 & 0 & 0 \\
\hline Liliopsida & Juncaceae & $\begin{array}{l}\text { Juncus scheuchzerioides } \\
\text { Gaudich. }\end{array}$ & $\mathrm{Hp}$ & $\mathrm{N}$ & 0 & 0 & 0,4 & 0 \\
\hline Liliopsida & Cyperaceae & $\begin{array}{l}\text { Schoenus } \\
\text { rhynchosporoides (Steud.) } \\
\text { Kük. }\end{array}$ & $\mathrm{Hp}$ & End & 0,4 & 0 & 0 & 0 \\
\hline Magnoliopsida & Escalloniaceae & Tribeles australis Phil. & sAr & End & 0,3 & 0 & 0 & 0 \\
\hline Bryopsida & Dicranaceae & $\begin{array}{l}\text { Dicranoloma imponens } \\
\text { (Mont.) Renauld }\end{array}$ & B & $\mathrm{N}$ & 0 & 0 & 0 & 0,2 \\
\hline Lecanoromycetes & Cladoniaceae & Cladonia sp. & $\mathrm{L}$ & $\mathrm{N}$ & 0 & 0 & 0 & 0,15 \\
\hline Magnoliopsida & Lentibulariaceae & $\begin{array}{l}\text { Pinguicula antarctica } \\
\text { Vahl }\end{array}$ & $\mathrm{Hp}$ & End & 0,13 & 0 & 0 & 0 \\
\hline Polypodiopsida & Blechnaceae & $\begin{array}{l}\text { Blechnum penna-marina } \\
\text { (Poir.) Kuhn }\end{array}$ & $\mathrm{Hp}$ & $\mathrm{N}$ & 0,1 & 0 & 0 & 0 \\
\hline Liliopsida & Poaceae & $\begin{array}{l}\text { Anthoxanthum redolens } \\
\text { (Vahl) P.Royen }\end{array}$ & $\mathrm{Hp}$ & $\mathrm{N}$ & 0,1 & 0 & 0 & 0 \\
\hline Magnoliopsida & Gunneraceae & $\begin{array}{l}\text { Gunnera magellanica } \\
\text { Lam. }\end{array}$ & $\mathrm{Hp}$ & $\mathrm{N}$ & 0 & 0 & 0,06 & 0 \\
\hline Bryopsida & Polytrichaceae & Polytrichadelphus sp. & B & $\mathrm{N}$ & 0 & 0 & 0,05 & 0 \\
\hline \multirow[t]{3}{*}{ Lecanoromycetes } & Acarosporaceae & $\begin{array}{l}\text { Endocena informis } \\
\text { Cromb. }\end{array}$ & Hep & $\mathrm{N}$ & 0 & 0,03 & 0 & 0 \\
\hline & & Nro. Total de Especies & & & 21 & 14 & 14 & 13 \\
\hline & & Cob. Total de Especies & & & 94,55 & 94,49 & 96,78 & 88,75 \\
\hline
\end{tabular}

\section{DISCUSIÓN}

La intensidad y esfuerzo de muestreo realizado en este estudio permitió incrementar el conocimiento de la riqueza y composición de las turberas no intervenidas por la cosecha de musgo Sphagnum en la región de Aysén. Se registró en total 30 especies, de ellas 24 spp. $(86 \%)$ corresponden a plantas vasculares, las que representan un 3\% de la flora vascular citada para la región de Aysén (Rodríguez \& Marticorena 2019). Por otra parte, es importante resaltar la ausencia de especies introducidas en las cuatro turberas estudiadas a diferencia de las turberas de la cuenca del río Baker y Pascua, en donde fue hallada Festuca rubra L. (Sandoval et al. 2016).

La baja riqueza y diversidad florística hallada en este estudio puede estar relacionada a

Tabla 3. Promedio $( \pm \mathrm{DE})$ de los índices de diversidad florística para las cuatro turberas de Sphagnum estudiadas.

Letras minúsculas distintas representan diferencias significativas $(p<0,05)$.

\begin{tabular}{lcccc}
\hline Parámetros comunitarios & T1 & T2 & T3 & T4 \\
\hline Shannon -Wiener (H') & $1,47 \pm 0,24^{\mathrm{b}}$ & $1,12 \pm 0,33^{\mathrm{a}}$ & $0,97 \pm 0,4^{\mathrm{a}}$ & $0,96 \pm 0,22^{\mathrm{a}}$ \\
Dominancia (D) & $0,31 \pm 0,08^{\mathrm{b}}$ & $0,38 \pm 0,11^{\mathrm{ab}}$ & $0,48 \pm 0,19^{\mathrm{b}}$ & $0,47 \pm 0,12^{\mathrm{b}}$ \\
Exponencial de Shannon (exp H') & $4,46 \pm 1,04^{\mathrm{b}}$ & $3,24 \pm 1,14^{\mathrm{a}}$ & $2,87 \pm 1,26^{\mathrm{a}}$ & $2,66 \pm 0,61^{\mathrm{a}}$ \\
Inverso de Gini-Simpson $\left(1 / 1-\lambda^{\prime}\right)$ & $0,69 \pm 0,08^{\mathrm{b}}$ & $0,62 \pm 0,11^{\mathrm{ab}}$ & $0,52 \pm 0,19^{\mathrm{a}}$ & $0,53 \pm 0,12^{\mathrm{a}}$ \\
\hline
\end{tabular}




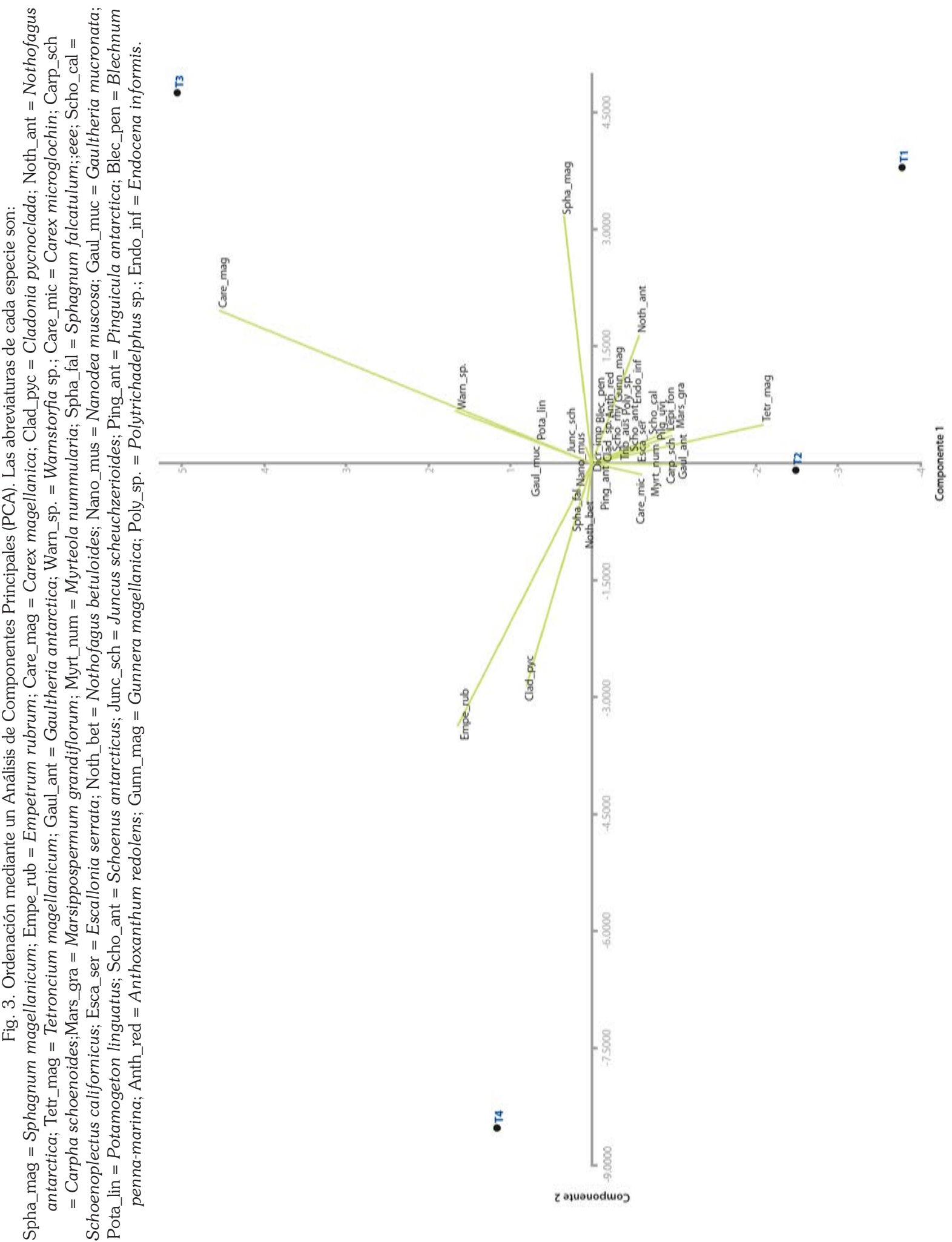


un gradiente altitudinal (Tabla 1), que determina condiciones climáticas como el incremento de las temperaturas y de las precipitaciones, junto con el aumento del nivel freático, favoreciendo este último el crecimiento del musgo Sphagnum, el cual a su vez afecta fuertemente las condiciones hidrológicas e hidroquímicas de las turberas. Estos resultados concuerdan con los hallazgos de otros estudios realizados, donde se ha documentado la influencia de ambas variables sobre la diversidad y composición florística en turberas (Van, 1995; van der Schaaf, 2002; Walker et al. 2017).

La diversidad efectiva, estimada a través del indice exponencial de entropía de Shannon (exp $\left.\mathrm{H}^{\prime}\right)$, es menor a la reportada para las turberas de Sphagnum de la cuenca de los ríos Baker y Pascua, donde el índice de Shannon-Wiener fue de $\left(H^{\prime}=2,038\right)$, esto es equivalente a (exp $H^{\prime}=$ $7,67)$. Este valor tan alto quizás se pueda deber a la intensa intervención antrópica de las comunidades vegetales en ambos sectores, la que ha favorecido la diversidad florística, en comparación a la cuenca del río Mayer. Por otra parte, las turberas de Sphagnum de la región de Magallanes también presentaron una mayor diversidad florística con un (exp H'= 4,68 $\pm 0,37$ ) (Domínguez et al. 2015), pero inferior a los resultados obtenidos por Sandoval y colaboradores (2016) y cercanos al valor más alto

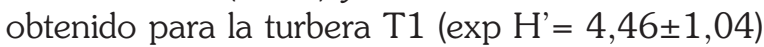
en este estudio. Tal vez, estas diferencias halladas responden a un factor climático de precipitación, el cual afecta a todas las turberas que forman parte de la cuenca del río Mayer. Las precipitaciones serían las responsables de mantener un nivel freático elevado, favoreciendo el crecimiento del Sphagnum magellanicum en ambientes anegados y afectando a las especies leñosas como Empetrum rubrum, en sitios con un alto nivel freático. Otra explicación podría deberse al aislamiento biogeográfico y a su historia natural, asociada a los continuos avances y retrocesos de los glaciares ocurridos en el pasado (Pisano, 1977). Esto podría explicar la baja riqueza y diversidad, como ha sido detectada en otros grupos taxonómicos, en similares latitudes, por ejemplo, en el Parque Nacional Bernardo O'Higgins (Muñoz-Escobar \& Jerez, 2017).

El análisis exploratorio realizado con PCA muestra un gradiente florístico en las turberas estudiadas. En el componente 1, el musgo
Sphagnum magellanicum puede ser considerado una especie indicadora de ambientes anegados, de acuerdo a lo propuesto por Pisano (1977), Teneb y Dollenz (2004), Kleinebecker y colaboradores (2008) para las turberas de Sphagnum en Magallanes. Estudios más recientes en restauración afirman que un nivel adecuado de agua de lluvia es un requisito importante para el crecimiento exitoso del musgo Sphagnum (Brust et al. 2018; Shi et al. 2020). Por otra parte, la especie subarbustiva Empetrum rubrum se encuentra en sentido opuesto al musgo Sphagnum, es decir, en ambientes más secos. Esto se debería a que este subarbusto no puede crecer en condiciones anóxicas y con un nivel de agua superficial, lo cual se ajusta a lo descrito en intervenciones mineras de turba en la región de Magallanes (Domínguez et al. 2012). Por otro lado, el componente 2 no está claro qué gradiente ambiental pueda estar representando, las especies segregadas mencionadas (Tetroncium magellanicum y Carex magellanica) crecen en turberas de Sphagnum y toleran los altos niveles freáticos; la única explicación plausible podría estar relacionada a la disponibilidad de oxígeno disuelto y la conductividad eléctrica, en los primeros $30 \mathrm{~cm}$ de la cubierta vegetal, la que se caracteriza por tener fluctuaciones respecto al nivel freático, generando variaciones en ambos parámetros ambientales. Sin embargo, esto debe ser evaluado con instrumentos.

Es importante tener en cuenta que la baja riqueza de especies y diversidad, hallada en este estudio, podría ser afectada por la extracción comercial de musgo Sphagnum. Por esta razón deben ser monitoreados los cambios en la composición y abundancia de las especies dominantes y raras, en este tipo de turberas. Esto, considerando que, al cosechar el musgo, se está dejando al descubierto una gran superficie susceptible a la disminución del nivel freático por la evaporación del agua y a la colonización por otras especies menos hidrófilas. De ahí la relevancia de dejar sitios sin cosechar que permitan o faciliten la regeneración natural del musgo Sphagnum.

La información entregada en este trabajo permite evaluar patrones y procesos comunitarios relacionados a la diversidad florística de las turberas de Sphagnum, lo que sirve de línea base y como referencia para futuros estudios de restauración ecológica. Lo anterior cobra 
importancia al considerar que actualmente existe una preocupación creciente y justificada, ya que las actividades humanas están provocando la pérdida irreparable de la diversidad a nivel global.

Finalmente, este estudio es una primera aproximación sobre el análisis de la magnitud de la diversidad florística en turberas de Sphagnum, entendiendo que es una propiedad y un indicador de la relevancia de las comunidades biológicas, y que los cambios en ella pueden utilizarse para justificar acciones de restauración de estos humedales y/o aumentar los niveles de protección del musgo Sphagnum magellanicum en Chile.

\section{AGRADECIMIENTOS}

Los autores quieren agradecer al programa: Bases Ambientales y Productivas para la Conservación y Uso Sustentable del musgo Sphagnum en la región de Aysén, financiado por el GORE de la región de Aysén del General Carlos Ibáñez del Campo y ejecutado por el INIA Tamel Aike. También se agradece a la Estancia Las Margaritas.

\section{LITERATURA CITADA}

Álvarez, M., San Martín, C., Novoa, C., Toledo, G., y Ramírez, C. (2010). Diversidad florística, vegetacional y de hábitats en el archipiélago de los Chonos (región de Aisén, Chile). Anales del Instituto de la Patagonia, 38(1), 35-56.

Armstrong, A., Waldron, S., Ostle, N., Richardson, H., y Whitaker, J. (2015). Biotic and abiotic factor interact to regulate peatland carbon cycling. Ecosystem, 18, 1395-1409.

Braun-Blanquet, J. (1979). Fitosociología. Bases para el estudio de las comunidades vegetales. Madrid: Blume Ediciones.

Brust, K., Krebs, M., Wahren, A., Gaudig, G., y Joosten, H. (2018). The water balance of a Sphagnum farming site in north-west Germany. Mires and Peat, 20(10), 1-12.

Caso, C., y Gil, M. A. (1988). The Gini-Simpson index of diversity: estimation in the stratified sampling. Communications in Statistics Theory and Methods, 17, 2981-2995.

Correa, M. N. (1969, 1971, 1978, 1984, 1985,
1988, 1999). Flora Patagónica I-VIII. Colección Científica INTA. Buenos Aires.

Domínguez, E., y Vega-Valdés, D. (Eds.). (2015). Funciones y servicios ecosistémicos de las turberas en Magallanes. Colección de libros INIA $N^{\circ} 33$. Instituto de Investigaciones Agropecuarias. Centro Regional de Investigación Kampenaike. Punta Arenas, Chile.

Domínguez, E., Bahamonde, N., y MuñozEscobar, C. (2012). Efectos de la extracción de turba sobre la composición y estructura de una turbera de Sphagnum explotada y abandonada hace 20 años, Chile. Anales del Instituto de la Patagonia, 40(2), 37-45.

Domínguez, E., Vega-Valdés, D., Dollenz, O., VillaMartínez, R., Aravena, J. C., Henríquez, J. M., y Muñoz-Escobar, C. (2015). Funciones y servicios ecosistémicos de las turberas en Magallanes. En: Florayvegetación de turberas de la Región de Magallanes. Colección de libros INIA N 33, pp. 149 - 195. Instituto de Investigaciones Agropecuarias. Centro Regional de Investigación Kampenaike, Punta Arenas, Chile.

García, N., y Luebert, F. (2008). Aporte al conocimiento de la flora y la vegetación del río Mosco, Villa O'Higgins, $48^{\circ} \mathrm{S}$ (Región de Aysén, Chile). Chloris Chilensis, Año 11, N². URL: http://www.chlorischile.cl

Hammer, Ø., Harper, D. A. T., y Ryan, P. D. (2001). PAST: Palaeontological statistics software package for education and data analysis. Palaeontología Electrónica, 4(1), 9.

Jost, L. (2006). Diversity and entropy. Oikos, 113(2), 363-375.

Jost, L. (2018). What do we mean by diversity? The path towards quantification. Mètode Science Studies Journal, 9, 55-61.

Jost, L., y González-Oreja, J. A. (2012). Midiendo la diversidad biológica: más allá del índice de Shannon. Acta zoológica lilloana, 56(1-2), 3-14.

Kleinebecker, T., Hölzer, N., y Vogel, A. (2008). South Patagonian ombrotrophic bog vegetation reflects biogeochemical gradients at the landscape level. Journal of Vegetation Science, 19, 151-160. 
Luebert, F., y Pliscoff, P. (2006). Sinopsis bioclimática y vegetacional de Chile. Santiago: Editorial Universitaria.

Maclaurin, J., y Sterelny, K. (2008). What is biodiversity? Chicago: The University of Chicago Press.

Moreno, C. E., Barragán, F., Pineda, E., y Pavón, N.P. (2011). Reanálisis de la diversidad alfa: alternativas para interpretar y comparar información sobre comunidades ecológicas. Revista mexicana de biodiversidad, 82(4), 1249-1261.

Muñoz-Escobar, C., y Jerez, V. (2017). Diversidad y composición de coleópteros del Parque Nacional Bernardo O'Higgins (región de Magallanes, Chile). Bosque, 38(2), 285297.

Noss, R. F. (1990). Indicators for Monitoring Biodiversity: A Hierarchical Approach. Conservation Biology, 4(4), 355-364.

Pisano, E. (1977). Fitogeografía de FuegoPatagonia chilena I. Comunidades vegetales entre las latitudes 52 y $56^{\circ} \mathrm{S}$. Anales del Instituto de la Patagonia, 8, 121-250.

Ramírez, C., San Martín, C., y Álvarez, I. (2007). Los turbales de Tortel (XI Región, Chile). Proyecto: FIA-PI-2004-1. Evaluación cuantitativa y cualitativa del musgo Sphagnum sp. como forma de conocer su uso, manejo y protección en la Comuna de Tortel, XI Región. Segundo Informe.

Rodríguez, R., y Marticorena, A. (Eds.). (2019). Catálogo de las plantas vasculares de Chile. Universidad de Concepción.

Rodríguez, R., Marticorena, A., y Teneb, E. (2008). Plantas vasculares de los ríos Baker y Pascua, Región de Aisén, Chile. Gayana Botánica, 65(1), 39-70.

San Martín, C., Montenegro, D., Pérez, Y., y Solís, J. L. (2014). Vegetación y flora leñosa de la comuna de Tortel (Región de Aisén, Chile). Agro Sur, 42(1), 15-29.

Sandoval, V., Ramírez, C., San Martín, C., Vidal, O., Álvarez, M., Marticorena, A., y Pérez, Y. (2016). Diversidad vegetal en las cuencas de los ríos Baker y Pascua (Aisén, Patagonia Chilena). Bosque, 37(2), 243-253.

Shannon, C. (1948). A mathematical theory of communication. The Bell System Technical
Journal, 27, 379-423.

Shi, X., Ricciuto, D.M., Thornton, P. E., Xu, X., Yuan, F., Norby, R. J., Walker, A. P., Warren, J., Mao, J., Hanson, P. J., Meng, L., Weston, D., y Griffiths, N. A. (2020). Modeling the hydrology and physiology of Sphagnum moss in a northern temperate bog. Biogeosciences. http://doi. org/10.5194/bg-2020-90

Simpson, E. H. (1949). Measurement of diversity. Nature, 163, 688.

Teneb, E., y Dollenz, O. (2004). Distribución espacial de la flora vascular, la humedad y el $\mathrm{pH}$ en un turbal de esfagno (Sphagnum magellanicum Brid.), Magallanes, Chile. Anales del Instituto de la Patagonia, 32, 5-12.

Teneb, E., Gómez, P., y González, M. (2008). Observaciones sobre la flora y vegetación de dos turberas en la región de Aisén, Patagonia Chilena. Gayana Botánica, 65(2), 229-232.

Van, B. N. (1995). How Sphagnum bogs down other plants. Trends in Ecology \& Evolution, 10, 270-275.

van der Maarel, E. (2007). Transformation of coverabundance values for appropriate numerical treatment Alternatives to the proposals by Podani. Journal of Vegetation Science, 18(5), 767-770.

van der Schaaf, S. (2002). Bog hydrology. In M. G. C. Schouten (Ed.), Conservation and restoration of raised bogs (pp. 54-109). Dept. of the Env. and Local Government; Staatsbosbeheer.

Villarroel, D., Henríquez, J., Domínguez, E., Silva, F., Martínez, M., y Báez, J. (2021). Funciones y servicios ecosistémicos de las turberas de Sphagnum en la región de Aysén. En: Distribución geográfica de turberas de Sphagnum en la región de Aysén. Colección de Libros INIA $N^{\circ} 41$, pp. 21-47. Instituto de Investigaciones Agropecuarias. Centro Regional de Investigación Tamel Aike, Coyhaique. Chile.

Walker, A. J., Carter, K. R., Gu, L., Hanson, P. J., Malhotra, A., Norby, R. J., Sebestyen, S. D., Wullschleger, S. D., y Weston, D. J. (2017). Biophysical drivers of seasonal variability in Sphagnum gross primary production 
in a northern temperate bog. Journal of Geophysical Research: Biogeosciences, 122, 1078-1097.

Yu, Z., Beilman, D. W., Frolking, S., MacDonald, G. M., Roulet, N. T., Camill, P., y Charman, D. J. (2011). Peatlands and Their Role in the Global Carbon Cycle. Earth and Space
Science News, 92, 97-108.

Zuloaga, F., Morrone, O., y Belgrano, M. (2020). Catálogo de las Plantas Vasculares del Cono Sur. Versión base de datos en sitio web del Instituto Darwinion, Argentina. URL: http:// www.darwin.edu.ar/Proyectos/ FloraArgentina/FA.asp: octubre 04, 2020. 